\title{
A real-time optimal inverse planning for Gamma Knife radiosurgery by convex optimization: description of the system and first dosimetry data
}

\author{
Marc Levivier, MD, PhD, IFAANS, ${ }^{1}$ Rafael E. Carrillo, PhD, ${ }^{2,3}$ Rémi Charrier, MSc, ${ }^{4}$ \\ André Martin, MSc, ${ }^{4}$ and Jean-Philippe Thiran, $\mathrm{PhD}^{2}$ \\ 1Department of Neurosurgery and Gamma Knife Center, Lausanne University Hospital, Lausanne; ${ }^{2}$ Signal Processing \\ Laboratory (LTS5), Ecole Polytechnique Fédérale de Lausanne (EPFL); ${ }^{3}$ CSEM SA, Neuchâtel; and ${ }^{4}$ Intuitive Therapeutics SA, \\ Saint-Sulpice, Switzerland
}

OBJECTIVE The authors developed a new, real-time interactive inverse planning approach, based on a fully convex framework, to be used for Gamma Knife radiosurgery.

METHODS The convex framework is based on the precomputation of a dictionary composed of the individual dose distributions of all possible shots, considering all their possible locations, sizes, and shapes inside the target volume. The convex problem is solved to determine the plan, i.e., which shots and with which weights, that will actually be used, considering a sparsity constraint on the shots to fulfill the constraints while minimizing the beam-on time. The system is called IntuitivePlan and allows data to be transferred from generated dose plans into the Gamma Knife treatment planning software for further dosimetry evaluation.

RESULTS The system has been very efficiently implemented, and an optimal plan is usually obtained in less than 1 to 2 minutes, depending on the complexity of the problem, on a desktop computer or in only a few minutes on a high-end laptop. Dosimetry data from 5 cases, 2 meningiomas and 3 vestibular schwannomas, were generated with IntuitivePlan. Results of evaluation of the dosimetry characteristics are very satisfactory and adequate in terms of conformity, selectivity, gradient, protection of organs at risk, and treatment time.

CONCLUSIONS The possibility of using optimal, interactive real-time inverse planning in conjunction with the Leksell Gamma Knife opens new perspectives in radiosurgery, especially considering the potential use of the full capabilities of the latest generations of the Leksell Gamma Knife. This approach gives new users the possibility of using the system for easier and quicker access to good-quality plans with a shorter technical training period and opens avenues for new planning strategies for expert users. The use of a convex optimization approach allows an optimal plan to be provided in a very short processing time. This way, innovative graphical user interfaces can be developed, allowing the user to interact directly with the planning system to graphically define the desired dose map and to modify on-the-fly the dose map by moving, in a very user-friendly manner, the isodose surfaces of an initial plan. Further independent quantitative prospective evaluation comparing inverse planned and forward planned cases is warranted to validate this novel and promising treatment planning approach.

https://thejns.org/doi/abs/10.3171/2018.7.GKS181572

KEYWORDS Gamma Knife; stereotactic radiosurgery; inverse planning; dosimetry; convex optimization

$\mathrm{T}$ HE Leksell Gamma Knife (LGK, Elekta AB) is a dedicated device for cranial radiosurgery using concomitant gamma rays emitted from ${ }^{60} \mathrm{Co}$ radiation sources focusing at an isocenter. This design provides unique dosimetry characteristics with a very steep gradient. ${ }^{10,13,17,18}$ Usually, multiple isocenters are used to shape the desired irradiation to the target volume with a high conformity and selectivity. Manual forward planning (i.e., the user places each isocenter at specific stereotactic co- ordinates) is currently the standard, most frequent way to plan dosimetry for Gamma Knife radiosurgery (GKRS) procedures. However, manual forward planning needs technical expertise and experience, especially with the newest generations of LGK Perfexion and LGK ICON. It also requires specific training and is time-consuming, and often provides suboptimal solutions in terms of indices, treatment time, etc., with respect to the capabilities and flexibility of LGK. Thus, an optimal inverse planning sys-

ABBREVIATIONS GKRS = Gamma Knife radiosurgery; GUI = graphical user interface; LGK = Leksell Gamma Knife; LGP = Leksell GammaPlan.

SUBMITTED June 1, 2018. ACCEPTED July 19, 2018.

INCLUDE WHEN CITING DOI: 10.3171/2018.7.GKS181572. 
tem would be helpful and of great support to exploit the full capabilities of the LGK. Moreover, because the current inverse planning methods use a nonconvex approach, the risk of trapping in local minima of the cost function usually precludes obtaining the optimal solution and limits the speed of convergence. When current inverse planning methods are used, the planning should be finalized in most instances by adjusting the shots manually.

Here, we report a new inverse planning approach, based on a fully convex framework to be used in conjunction with Leksell GammaPlan (LGP) for LGK Perfexion and LGK ICON (all Elekta AB).

\section{Methods}

\section{Inverse Planning Software Principles}

The general principles of our convex framework are the following:

1) Based on the target volume definition, we precompute a dictionary composed of the individual dose distributions of all possible shots, considering all their possible locations, sizes, and shapes inside the target volume. To do this efficiently, we use an optimized implementation of the TMR (tissue-maximum ratio) 10 dose calculation formula, i.e., the reference formula used for dose calculation in LGP. ${ }^{6}$

2) Based on the desired dose in the target volume and potential additional constraints, we then solve a convex problem to determine the plan, i.e., which shots and with which weights, that will actually be used, considering a sparsity constraint on the shots to fulfill the constraints while minimizing the beam-on time.

This framework has been developed on the basis of our previous work using convex optimization approaches. ${ }^{1,3}$ It has several unique features in comparison with other existing approaches. First, it is globally convex, i.e., it ensures that a global optimum of the problem is found. Second, although the problem can be very large (i.e., the size of the dictionary can be extremely large), it can be efficiently parallelized and implemented using the latest, most efficient algorithms in convex optimization, such as in our case, a primal-dual convex optimization approach..$^{12}$ Third, the framework is very flexible, which allows different cost functions and sparsity constraints to be used. For instance, $\mathrm{L}_{0}$ or $\mathrm{L}_{1}$ norm minimization can be used to enforce a minimal number of shots and therefore a minimal beam-on time. Other formulations are also possible, such as a definition of the dictionary, not in terms of shots (i.e., number, possible locations, sizes, and geometry) but in terms of beams emitted by the different sectors, controlled by a structured sparsity constraint, which is actually what we have implemented in the version of the method used to generate the results presented hereafter.

The system is called IntuitivePlan and is developed by Intuitive Therapeutics SA.

\section{Generating a Plan in IntuitivePlan and Using the Data in LGP}

The workflow for using the inverse planning system in conjunction with LGP is illustrated in Fig. 1, and is as follows:
1) A patient file is created in LGP and includes all the mandatory steps needed to allow for shot placement (importing DICOM images and defining the stereotactic reference system, the fixation device and head contour, the target[s] and, if applicable, organ[s] at risk, and/ or other volume[s] of interest; creating a plan; selecting the dose prescription).

2) The data generated and included in the 2 following files are exported: the "Patient_DICOM-RT" file, using the "Export DICOM" function, and the "Patient.lgp" file, using the "Patient management-Export" function of LGP. These 2 files are used in our convex framework for inverse planning, according to the principles described above.

3) The data of the 2 files are then imported and opened in the IntuitivePlan software environment that runs on a separate computer. This will allow the patient's CT and/or MR images and appropriate contours, such as the skull, target volume(s), and organ(s) at risk, to be displayed. Appropriate constraints (i.e., minimum dose to the target[s] and maximum dose to organ[s] at risk, if any) are then defined by the user. The system will then prompt the user to adjust priorities for the selectivity, the gradient index, and the maximum dose. These parameters are used to optimize the beam-on time and the gradient, as well as to set a range for the isodose prescription. The plan is then automatically generated and displayed (Fig. 2 upper). If additional constraints are desired and defined, a new plan is generated and displayed (Fig. 2 lower). Further possibilities for the user to manipulate the plan directly and interactively in a very user-friendly manner (i.e., by moving directly the isodose lines with the mouse) are under development and are presented in the Discussion section and will be available in the commercialized version of the software.

4) The data of the dosimetry planning generated in IntuitivePlan are saved in a text file that contains the position (i.e., stereotactic coordinates), sector characteristics, and weight of each isocenter, as well as the maximum dose (which sets the percentage of the isodose prescription, according to the desired dose prescription). This text file is used to import the shots in LGP, using the "import" function in the shot window of LGP.

5) The user and team can then evaluate and validate the proposed plan in LGP as usual, and if appropriate, compare it with other dosimetry planning for the same patient, and eventually decide to use this plan for LGK treatment.

\section{Dosimetry Evaluation}

In order to evaluate the workflow between LGP and the inverse planning software in a clinical dosimetry context, we used anonymized data of 5 cases treated with manual forward planning. There were 2 meningiomas and 3 vestibular schwannomas. The data were processed as described above and the plans were displayed in LGP.

Also, courtesy of Ian Paddick (Physicist, London, United Kingdom), data from some of the United Kingdom benchmark cases were processed and analyzed in IntuitivePlan. The results of these multicenter benchmark 


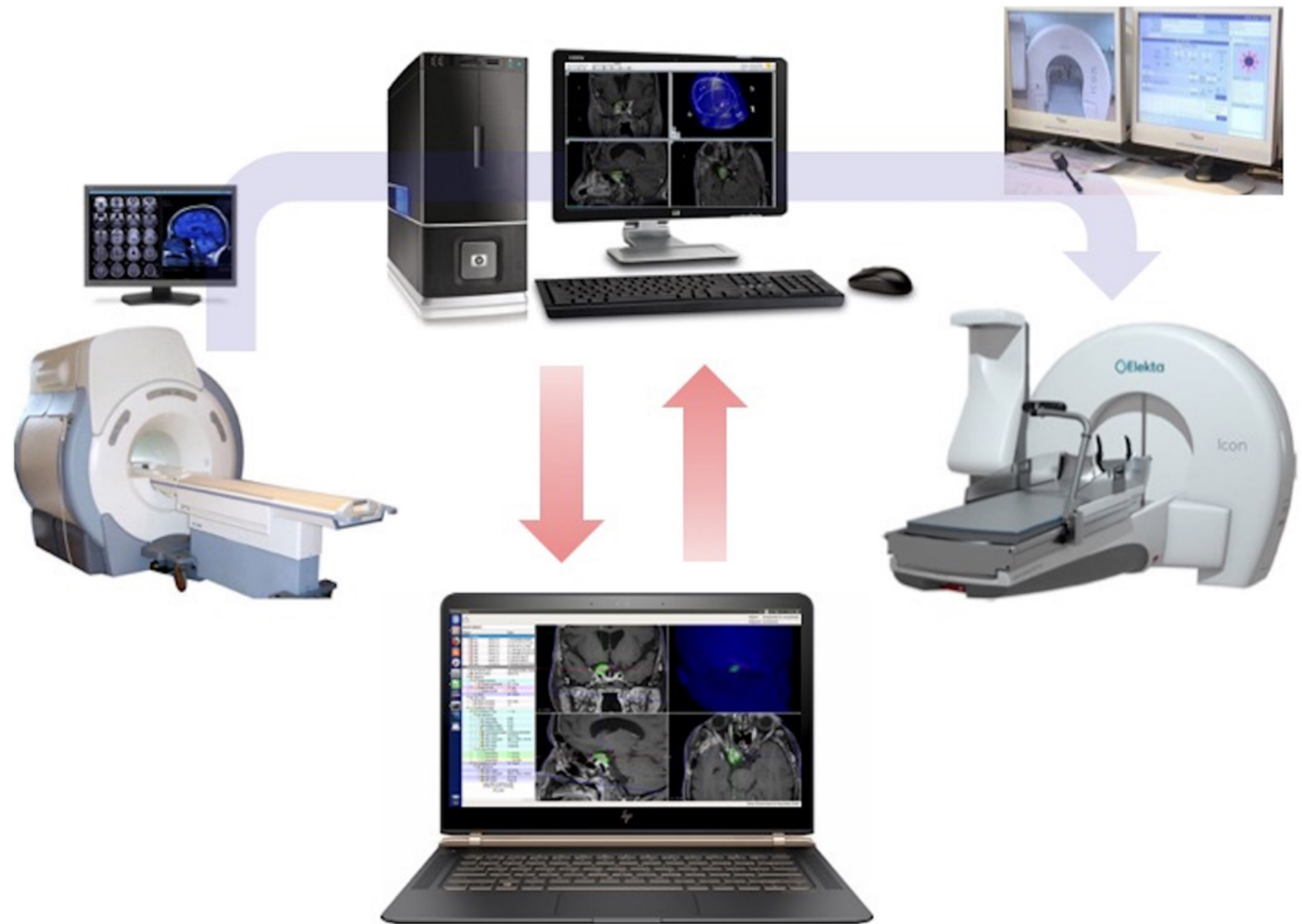

FIG. 1. Schematic of the workflow using the inverse planning system in conjunction with LGP. In a regular Gamma Knife workflow (gray arrow), images will be transferred to the computer running LGP, in which all the dosimetry planning steps will be performed. After its validation, the planning is exported to the Gamma Knife console for treatment. When using IntuitivePlan (red arrows), a patient file including all mandatory steps needed to allow for shot placements is created in LGP. The data are then imported and opened in the IntuitivePlan software environment that runs on a separate computer. The data of the dosimetry planning generated in IntuitivePlan are transferred to LGP, using the "import" function in the shot window of LGP. The user can then evaluate this plan in LGP as usual, and, if appropriate, compare it with other dosimetry planning for the same patient, and eventually decide to validate and use this plan for treatment as with the regular Gamma Knife workflow. Images of the LGK treatment console (upper right) and of the LGK ICON (middle right) are courtesy of Elekta AB.

planning studies have been published recently for multiple brain metastases ${ }^{4}$ and for benign brain tumors. ${ }^{5}$

\section{Results}

Inverse Planning Software System

The system has been very efficiently implemented and an optimal plan can be obtained usually in less than 1 to 2 minutes, depending on the complexity of the problem, on a desktop computer equipped with 2 high-end graphics processing units, or in only a few minutes on a high-end laptop. The specific graphical user interface (GUI) that has been developed for the system is shown in Fig. 2. The entire system allows production of optimal plans that can be exported to LGP, and thus seamlessly incorporated in the clinical workflow for GKRS.

Currently, the system works efficiently as a stand-alone solution (Fig. 1). The possibility of integrating this new soft- ware into the current planning system(s) for LGK would be of interest, but is outside the scope of the present work.

\section{Generating and Using the Data of the Inverse Planning Software in LGP}

For the 5 cases, the necessary data from LGP could be exported in IntuitivePlan and used for inverse planning. The data of the dose plans generated in IntuitivePlan were then imported and displayed in LGP. Dosimetry characteristics of the 5 plans generated with IntuitivePlan are shown in Table 1. The corresponding dosimetry characteristics of the manual forward plans that were actually used to treat the patients are also shown in Table 1.

\section{Discussion}

A typical GKRS intervention consists of a planning phase and a delivery phase. In the planning phase, each 

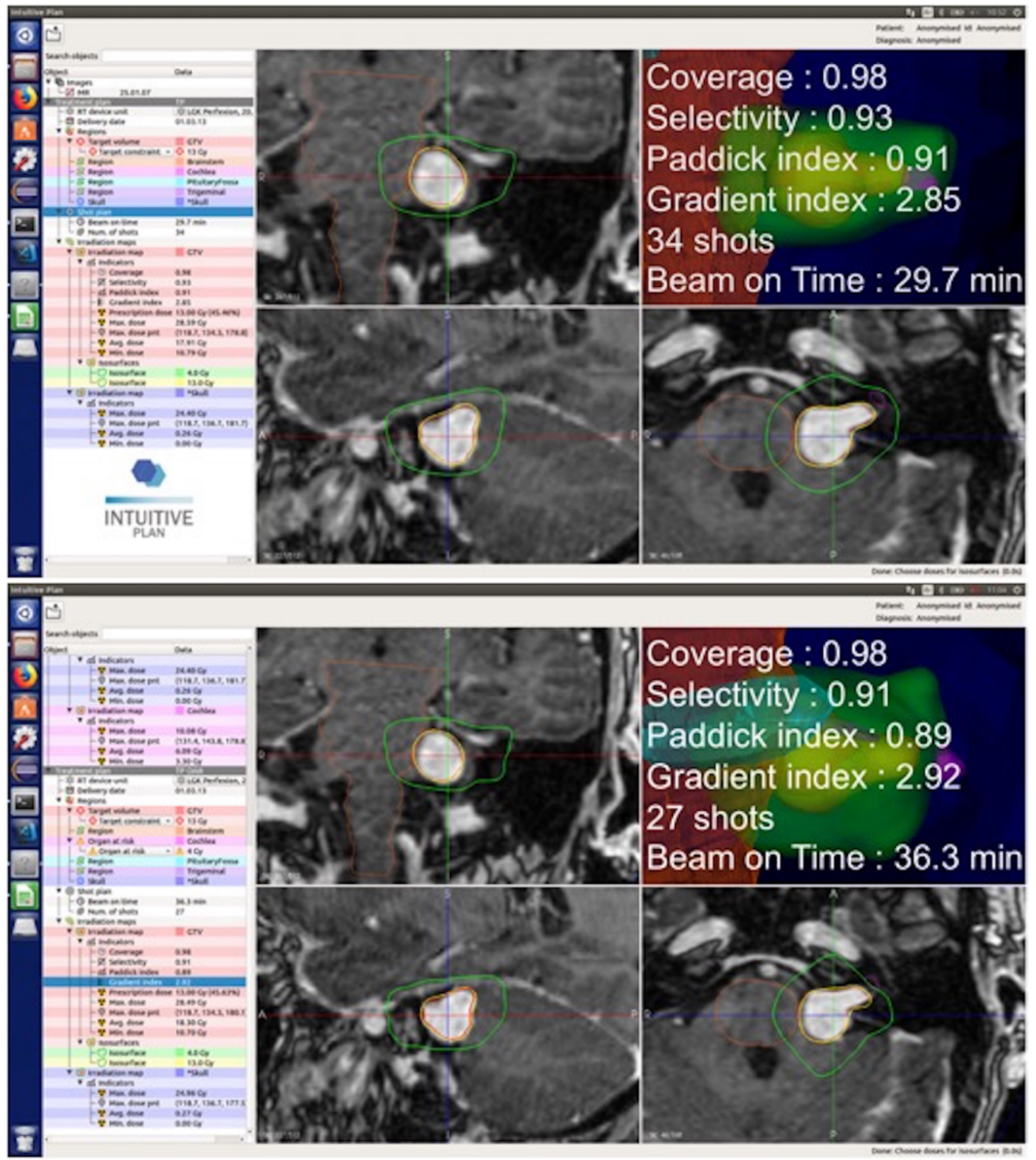

FIG. 2. Screen shots of the GUI of IntuitivePlan. A vestibular schwannoma from one of the United Kingdom benchmark cases (courtesy of lan Paddick), for which the target volume (red line) and the organs at risk (orange line, brainstem; purple line, cochlea) were preexisting, is tested in our inverse planning system. Upper: Only the prescription dose for target volume (13 Gy, yellow line) is used as a constraint. In the generated plan, the cochlea receives a dose higher than 4 Gy (green line). Lower: An additional constraint of a maximum of 4 Gy has been added for the cochlea, generating a new plan. The characteristics of each plan are displayed in the upper right of each panel. 


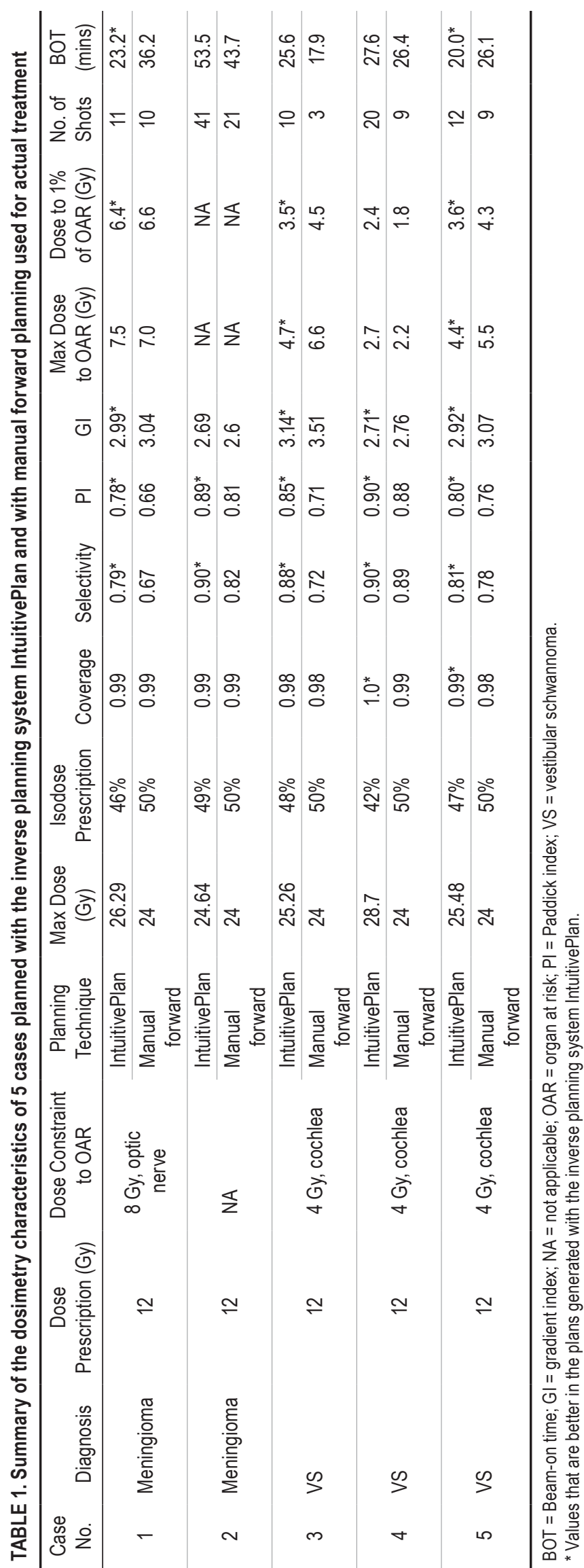

patient's treatment plan is developed by a neurosurgeon working in conjunction with a radiation oncologist and a physicist. According to the most widely used planning procedure, referred to as forward planning, they determine, through an iterative process of trial and error, the number and location of shots, along with their size, shape, and weight. When the treatment volume is small, the treatment plan may only require 1 or 2 shots. The planning process, however, becomes more complex for both irregularly shaped and larger target volumes. For these cases, the complexity of the treatment planning process makes it difficult to take full advantage of the powerful capabilities of the LGK. This is especially true in the latest versions, LGK Perfexion and LGK ICON, where the cobalt sources are grouped in sectors that align concomitantly with collimators of the same size. Each individual sector can be aligned with a specific collimator size (composite shot) or can be blocked. GKRS thus requires a critical stage of planning in order to create the clinically acceptable dosimetry based on the location and dose to be delivered to the target, and to the possible organs at risk. The technical parameters to be set for creating a dose distribution adapted to the desired irradiation map are mainly the number of the irradiation focal points (isocenters), the location of those isocenters, the size and shape of the collimation of the irradiation beams (including the possibility to create composite shots), and the weight of the different irradiation shots.

Thus, the current manual procedure for the planning step is relatively complex, tedious, unintuitive, and slow. The duration of the forward planning procedure decreases the productivity and increases the cost of every treatment. Moreover, its quality depends essentially on the experience of the user. Acquiring this experience requires a long training period in one of the few reference centers in the world. To help the user, automatic inverse planning systems have been proposed. The planning is "inverse" in that, based on the knowledge of the target region properties (e.g., from CT or MR images), the operator prescribes a certain dose distribution within the target region and/or certain dose constraints. An automatic inverse planning system finds a set of parameters, resulting in treatment planning that is as close as possible to the desired dose distribution.

Inverse planning is thus typically defined as an optimization problem, where the technical parameters are automatically searched to minimize a cost function measuring the difference between the desired dose distribution and that actually achieved. But, as pointed out earlier by $\mathrm{Wu}$ et al., ${ }^{19}$ the main challenge in LGK inverse planning is the large dimension of the search. Hence, planning or placing these shots is a combinatorial optimization process that is computationally expensive by nature. There has been limited previous research in LGK treatment optimization due to both the large dimensionality of the optimization problem and the impracticality of delivering complex treatments clinically with older LGK models. . $^{\text {-9 }}$

Considering the technical characteristics of LGK Perfexion and LGK ICON, the problem is even more acute. Indeed, the combination of 8 sectors (blocked or not) and 3 collimator sizes makes the number of possible configurations for one single shot as large as 4 to the power of 
8 (i.e., 65,536). In this context, very few inverse planning systems have been developed. Elekta itself proposes an inverse planning system in LGP, but this system is generally considered as suboptimal, providing at most a reasonable, first approximation dose plan. Particularly in cases in which eloquent structures are at risk, experience and user-based optimization are still required to achieve an acceptable dose plan. ${ }^{16}$ One particularly attractive avenue to solve this complex optimization problem is to exploit convex optimization techniques, which have the intrinsic property of providing the optimal solution, as long as the function to be optimized can be expressed as convex. ${ }^{2} \mathrm{~A}$ first step in this direction has been taken by Ghobadi et al. ${ }^{11}$ In that paper, the treatment plan was obtained using a 2-step approach. First, a hybrid grassfire and spherepacking algorithm was used to obtain shot positions (isocenters) based on the geometry of the target to be treated. For the selected isocenters, a sector duration optimization (SDO) model was used to optimize the duration of radiation delivery from each collimator size from each individual source bank. The SDO model is convex and is solved using a projected gradient algorithm. Although interesting, this approach is not globally convex and is therefore suboptimal, since a first step is used to predetermine the isocenter locations based on the target geometry only and ignores the large variety of possible shot geometries to define those isocenters.

As mentioned above, our approach is unique in the sense that, to the best of our knowledge, it is the first system that defines and solves the entire inverse planning problem in a convex optimization framework, i.e., optimizing both the shot or beam locations and weights together and not in a sequential manner. This system will thus span a much larger space of possible solutions and find the optimal one. Moreover, since convex optimization is intrinsically well suited for parallel implementation, an optimal plan is provided in a very short processing time. This makes it possible to develop innovative GUIs, namely in allowing the user to interact directly with the planning system to graphically define the desired dose map but also, and most importantly, to modify on-the-fly the dose map proposed by a first run of the system. Indeed, in such a GUI, the user could interactively modify the requested dose map by moving, in a very user-friendly manner, i.e., directly with the mouse, the isodose surfaces of an initial plan. This will allow users, for instance, to impose more constraints on some organs and regions at risk or relax other unnecessary constraints, avoiding the tedious tasks of contouring new regions of interest as would be done with other systems, and/or the need to change some shot placement manually to finalize the desired planning results. Thus, with this approach, the user will not need to manipulate the planning parameters himself or herself (i.e., placement and weight of shots) but will be able to directly move the isodose lines interactively while the system is recalculating a new plan, ultimately in real time. This interactive real-time inverse planning system would ultimately lead to the definition of plans that are optimal both technically and clinically. Such an interactive real-time approach is currently under implementation for integration into IntuitivePlan. Also, since the dosimetry requirements might be different with respect to the disease process (e.g., benign tumors vs metastases) and the number of lesions (especially multiple metastases), different presetting priorities for the selectivity, the gradient index, and the maximum dose are envisioned.

The possibility of using optimal interactive real-time inverse planning in conjunction with LGP opens new perspectives for the use of GKRS. Long-term users may find it advantageous to reduce tedious planning time while still being able to interact with the dosimetry results in an intuitive manner, relaxing some constraints and/or increasing others, based on their clinical experience and radiobiological knowledge. In addition to the possibility of directly manipulating lower isodose lines in order to shape the gradient in a desired way, this approach could also be used to manipulate bridging doses when treating nearby multiple lesions, or even to manipulate higher isodoses (i.e., the so-called hot spots) into the treated target volumes. Moreover, the system is designed to fulfill the constraints while minimizing the beam-on time. Thus, in addition to the dramatic reduction in planning time, the system will also allow users to reduce the treatment time. So, all in all, the use of IntuitivePlan could favorably decrease the total time of a GKRS procedure, which may have a positive economic impact on the workflow of Gamma Knife centers. For new users, the potential to use an optimal inverse planning approach will permit easier and quicker access to good-quality plans with a shorter technical training period. Of course, this does not replace acquiring the appropriate knowledge and training in radiosurgery in order to understand the proper management of patients who would benefit from this approach. Ideally, it means that the dosimetry plans provided by the inverse planning should, at least, match the quality of those of experienced manual planners. The dosimetry data that we have generated so far are encouraging in that respect. The quality of the plans meets the expected standards that are used in clinical plans, including when using well-recognized indices and criteria. ${ }^{14,15}$ However, the proof of concept reported here and the ability to generate good qualitative plans do not prevent comparing quantitatively inverse planned and forward planned cases with a series of objective criteria (e.g., gradient index, coverage, conformality, V12, V10, V8, total treatment time, and meeting dose constraints). We believe that such studies should be performed independently by groups interested in testing the system, in order to avoid any bias and conflict of interest. As a first step in that direction, a recent independent retrospective study, comparing the manual plans of an expert LGK user with the plans generated by IntuitivePlan in a consecutive series of 30 vestibular schwannomas, has shown comparable results in terms of conformity, Paddick index, gradient index, and treatment time, while the selectivity index was significantly better in the inverse plans compared to the manual plans performed by the expert (Regis J, Merly L: Is Intuitive Therapy plan competing with dose planning elaborated by an expert? A comparative study in vestibular schwannomas, presented at the 19th Leksell Gamma Knife Society Meeting, Dubai, United Arab Emirates, March 4-8, 2018). Further independent prospective studies evaluating IntuitivePlan quantitatively in different disease conditions are warranted and are planned to validate this novel and promising treatment planning approach. 


\section{Conclusions}

We have developed a new inverse planning approach based on a fully convex framework to be used in conjunction with LGP for LGK Perfexion and LGK ICON. The system, called IntuitivePlan, allows an optimal plan to be obtained in less than 1 to 2 minutes, depending on the complexity of the problem, and the results can then be easily imported and integrated into LGP. The first dosimetry data of plans generated with IntuitivePlan are qualitatively of high standard, and a first independent retrospective study has provided results comparing favorably to the forward manual plans performed by the expert. The possibility of using an optimal interactive real-time inverse planning in conjunction with LGP opens new perspectives for the use of GKRS. The use of a convex optimization approach allows an optimal plan to be provided in a very short processing time. This makes it possible to develop innovative GUIs, namely allowing the user to interact directly with the planning system in order to define graphically the desired dose map and to modify on-the-fly the dose map by moving, in a very user-friendly manner, directly with the mouse, the isodose surfaces of an initial plan. Further independent quantitative prospective evaluation comparing inverse planned and forward planned cases is warranted to validate this novel and promising treatment planning approach.

\section{References}

1. Auría A, Carrillo R, Thiran JP, Wiaux Y: Tensor optimisation for optical-interferometric imaging. Mon Not R Astron Soc 437:2083-2091, 2014

2. Boyd S, Vandenberghe L (eds): Convex Optimization. Cambridge, UK: Cambridge University Press, 2004

3. Daducci A, Dal Palù A, Lemkaddem A, Thiran JP: COMMIT: Convex optimization modeling for microstructure informed tractography. IEEE Trans Med Imaging 34:246257, 2015

4. Eaton DJ, Lee J, Paddick I: Stereotactic radiosurgery for multiple brain metastases: results of multicenter benchmark planning studies. Pract Radiat Oncol 8:e212-e220, 2018

5. Eaton DJ, Lee J, Patel R, Millin AE, Paddick I, Walker C: Stereotactic radiosurgery for benign brain tumors: results of multicenter benchmark planning studies. Pract Radiat Oncol [epub ahead of print], 2018

6. Elekta AB: A New TMR Dose Algorithm in Leksell GammaPlan. Technical Report 1021357.00. Stockholm: Elekta $\mathrm{AB}, 2011$

7. Ferris MC, Lim J, Shepard DM: An optimization approach for radiosurgery treatment planning. SIAM J Optim 13:921937, 2003

8. Ferris MC, Lim J, Shepard DM: Radiosurgery treatment planning via nonlinear programming. Ann Oper Res 119:247-260, 2001

9. Ferris MC, Shephard DM: Optimization of Gamma Knife radiosurgery, in Du DZ, Pardolas P, Wang J (eds): Discrete Mathematical Problems with Medical Applications. DIMACS Series in Discrete Mathematics and Theoretical Computer Science. Providence: American Mathematical Society, 2000, Vol. 55, pp 27-44

10. Gevaert T, Levivier M, Lacornerie T, Verellen D, Engels B, Reynaert N, et al: Dosimetric comparison of different treatment modalities for stereotactic radiosurgery of arteriovenous malformations and acoustic neuromas. Radiother Oncol 106:192-197, 2013

11. Ghobadi K, Ghaffari HR, Aleman DM, Jaffray DA, Ruschin
M: Automated treatment planning for a dedicated multisource intracranial radiosurgery treatment unit using projected gradient and grassfire algorithms. Med Phys 39:31343141,2012

12. Komodakis N, Pesquet JC: Playing with duality: an overview of recent primal-dual approaches for solving large-scale optimization problems. IEEE Trans Signal Process 32:31-54, 2015

13. Lindquist $\mathrm{C}$, Paddick I: The Leksell Gamma Knife Perfexion and comparisons with its predecessors. Neurosurgery 61 (3 Suppl):130-141, 2007

14. Paddick I: A simple scoring ratio to index the conformity of radiosurgical treatment plans. Technical note. J Neurosurg 93 (Suppl 3):219-222, 2000

15. Paddick I, Lippitz B: A simple dose gradient measurement tool to complement the conformity index. J Neurosurg 105 Suppl:194-201, 2006

16. Schlesinger DJ, Sayer FT, Yen CP, Sheehan JP: Leksell GammaPlan version 10.0 preview: performance of the new inverse treatment planning algorithm applied to Gamma Knife surgery for pituitary adenoma. J Neurosurg 113 Suppl:144-148, 2010

17. Tuleasca C, Negretti L, Faouzi M, Magaddino V, Gevaert T, von Elm E, et al: Radiosurgery in the management of brain metastasis: a retrospective single-center study comparing Gamma Knife and LINAC treatment. J Neurosurg 128:352361, 2018

18. Wu A: Physics and dosimetry of the gamma knife. Neurosurg Clin N Am 3:35-50, 1992

19. Wu QJ, Chankong V, Jitprapaikulsarn S, Wessels BW, Einstein DB, Mathayomchan B, et al: Real-time inverse planning for Gamma Knife radiosurgery. Med Phys 30:2988-2995, 2003

\section{Disclosures}

Dr. Levivier: scientific advisor and board member of Intuitive Therapeutics SA. Mr. Charrier: employee of and ownership in Intuitive Therapeutics SA. Mr. Martin: employee and board member of Intuitive Therapeutics SA. Dr. Thiran: scientific advisor and board member of Intuitive Therapeutics SA.

The system presented here was initially developed in the Signal Processing Laboratory LTS5 of the Ecole Polytechnique Fédérale de Lausanne (EPFL), Lausanne, Switzerland, with the financial support of Intuitive Therapeutics SA, Saint-Sulpice, Switzerland, and then transferred to Intuitive Therapeutics SA for final product development and industrialization.

\section{Author Contributions}

Conception and design: Levivier, Carrillo, Thiran. Acquisition of data: Levivier, Carrillo, Charrier, Thiran. Analysis and interpretation of data: all authors. Drafting the article: Levivier, Thiran. Critically revising the article: Carrillo, Charrier, Martin, Thiran. Reviewed submitted version of manuscript: all authors. Approved the final version of the manuscript on behalf of all authors: Levivier. Administrative/technical/material support: Charrier, Martin. Study supervision: Levivier, Thiran.

\section{Supplemental Information \\ Previous Presentations}

A portion of this work was presented at the 19th Leksell Gamma Knife Society Meeting, Dubai, United Arab Emirates, March 4-8, 2018.

\section{Correspondence}

Marc Levivier: Department of Neurosurgery \& Gamma Knife Center, Lausanne University Hospital, Lausanne, Switzerland. marc.levivier@chuv.ch. 